\title{
Front Matter: Volume 10721
}

, "Front Matter: Volume 10721," Proc. SPIE 10721, Active Photonic Platforms X, 1072101 (17 October 2018); doi: 10.1117/12.2516829

SPIE Event: SPIE Nanoscience + Engineering, 2018, San Diego, California, United SPIE. States 


\title{
PROCEEDINGS OF SPIE
}

\section{Active Photonic Platforms X}

\author{
Ganapathi S. Subramania \\ Stavroula Foteinopoulou \\ Editors
}

19-23 August 2018

San Diego, California, United States

Sponsored and Published by

SPIE 
The papers in this volume were part of the technical conference cited on the cover and title page. Papers were selected and subject to review by the editors and conference program committee. Some conference presentations may not be available for publication. Additional papers and presentation recordings may be available online in the SPIE Digital Library at SPIEDigitalLibrary.org.

The papers reflect the work and thoughts of the authors and are published herein as submitted. The publisher is not responsible for the validity of the information or for any outcomes resulting from reliance thereon.

Please use the following format to cite material from these proceedings:

Author(s), "Title of Paper," in Active Photonic Platforms X, edited by Ganapathi S. Subramania, Stavroula Foteinopoulou, Proceedings of SPIE Vol. 10721 (SPIE, Bellingham, WA, 2018) Seven-digit Article CID Number.

ISSN: 0277-786X

ISSN: 1996-756X (electronic)

ISBN: 9781510620131

ISBN: 9781510620148 (electronic)

Published by

SPIE

P.O. Box 10, Bellingham, Washington 98227-0010 USA

Telephone +1 3606763290 (Pacific Time) · Fax +1 3606471445

SPIE.org

Copyright (C) 2018, Society of Photo-Optical Instrumentation Engineers.

Copying of material in this book for internal or personal use, or for the internal or personal use of specific clients, beyond the fair use provisions granted by the U.S. Copyright Law is authorized by SPIE subject to payment of copying fees. The Transactional Reporting Service base fee for this volume is $\$ 18.00$ per article (or portion thereof), which should be paid directly to the Copyright Clearance Center (CCC), 222 Rosewood Drive, Danvers, MA 01923. Payment may also be made electronically through CCC Online at copyright.com. Other copying for republication, resale, advertising or promotion, or any form of systematic or multiple reproduction of any material in this book is prohibited except with permission in writing from the publisher. The CCC fee code is 0277$786 \mathrm{X} / 18 / \$ 18.00$.

Printed in the United States of America.

Publication of record for individual papers is online in the SPIE Digital Library.

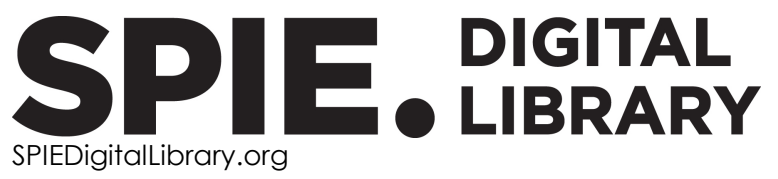

Paper Numbering: Proceedings of SPIE follow an e-First publication model. A unique citation identifier (CID) number is assigned to each article at the time of publication. Utilization of CIDs allows articles to be fully citable as soon as they are published online, and connects the same identifier to all online and print versions of the publication. SPIE uses a seven-digit CID article numbering system structured as follows:

- The first five digits correspond to the SPIE volume number.

- The last two digits indicate publication order within the volume using a Base 36 numbering system employing both numerals and letters. These two-number sets start with $00,01,02,03,04$, 05, 06, 07, 08, 09, 0A, OB ... 0Z, followed by 10-1Z, 20-2Z, etc. The CID Number appears on each page of the manuscript. 


\title{
Contents
}

\author{
$\checkmark \quad$ Authors \\ vii Conference Committee \\ xi Introduction
}

NOVEL NONLINEAR PHOTONIC PHENOMENA II

1072106 Epsilon-near-zero plasmonic waveguides to enhance nonlinear coherent light-matter interactions [10721-5]

PHASE-CHANGE MATERIALS FOR TUNEABLE PHOTONICS

$10721 \mathrm{OH} \quad$ RGB tunable color filters using germanium telluride [10721-16]

10721 ol Using phase-change materials to switch the direction of reflectionless light propagation in nonPT-symmetric structures (Invited Paper) [10721-17]

TWISTED LIGHT: ORBITAL ANGULAR MOMENTUM AND VORTEX STATES

$107210 X \quad$ Symmetry principles for engaging the orbital angular momentum of structured light [10721-33]

HARNESSING LIGHT-MATTER INTERACTION I

10721 IG Efficient modeling techniques in nanophotonics [10721-52]

PHOTONIC MEMORIES AND NEUROMORPHIC COMPUTING

$107211 \mathrm{M}$ Integrated neuromorphic photonics (Invited Paper) [10721-58] 
ADDRESSING MODELING CHALLENGES IN EMERGING AND ACTIVE PHOTONIC SYSTEMS

10721 IV Computational plasmonics with applications to bulk and nanosized systems (Invited Paper) [10721-67]

NEW MATERIALS FOR PHOTONICS: FROM "NATURE'S LAB" TO ADVANCED FABRICATION

1072122 Design principles for nanoparticle based photonic crystals [10721-75]

HARNESSING LIGHT-MATTER INTERACTION II

$1072128 \quad$ Strong light-matter coupling in a quantum metasurface [10721-81]

POSTER SESSION

$107212 B \quad$ Role of interface defect in hot carriers extraction at graphene-metal contact [10721-84]

$107212 \mathrm{C} \quad$ Optimal control of quantum systems with ultrashort laser pulses and non-adiabatic interactions [10721-85]

$107212 \mathrm{D}$ Analysis of the effect of graphene integration on the coupling condition in microresonator [10721-86]

$107212 \mathrm{E} \quad$ Experimental noise-free information recovery via reference beam encryption [10721-87] 


\section{Authors}

Numbers in the index correspond to the last two digits of the seven-digit citation identifier (CID) article numbering system used in Proceedings of SPIE. The first five digits reflect the volume number. Base 36 numbering is employed for the last two digits and indicates the order of articles within the volume. Numbers start with 00, 01, 02, 03, 04, 05, 06, 07, 08, 09, 0A, 0B...0Z, followed by 10-12, 20-2Z, etc.

Andrews, David L., OX

Argyropoulos, Christos, 06

Barrera Ramírez, John Fredy, 2E

Felbacq, Didier, 28

Ferreira de Lima, Thomas, 1M

Guo, L. J., $\mathrm{OH}$

Huang, Yin, $\mathrm{Ol}$

Hughes, Stephen, $1 G$

Jafari, Mohsen, $\mathrm{OH}$

Jang, Min Seok, 2B

Jaramillo Osorio, John Alexis, 2E

Joshi, Swati, 2D

Kamandar Dezfouli, Mohsen, IG

Kim, Ju Yeong, 2B

Kumar Kaushik, Brajesh, 2D

Li, Ying, 06

Lin, Haixin, 22

Mehay, Timothy, $1 \mathrm{~V}$

Menabde, Sergey G., 2B

Min, Changjun, $\mathrm{Ol}$

Min, Sung Yoon, 2B

Mira-Agudelo, Alejandro, 2E

Mirkin, Chad A., 22

Montoya, Santiago, 2E

Nahmias, Mitchell A., $1 \mathrm{M}$

Peng, Hsuan-Tung, $1 \mathrm{M}$

Prucnal, Paul R., 1M

Quandt, Alexander, IV

Rais-Zadeh, Mina, $\mathrm{OH}$

Ramírez Quiceno, Mariana, 2C

Rousseau, Emmanuel, 28

Sanz-Vicario, José Luis, 2C

Schatz, George C., 22

Shastri, Bhavin J., $1 \mathrm{M}$

Shen, Yuecheng, 0 I

Sun, Lin, 22

Tait, Alexander N., $1 \mathrm{M}$

Torroba, Roberto, 2E

Vasco, Juan Pablo, $1 \mathrm{G}$

Velez Zea, Alejandro, 2E

Veronis, Georgios, 01

Warmbier, Robert, IV 
Proc. of SPIE Vol. 10721 1072101-6

Downloaded From: https://www.spiedigitallibrary.org/conference-proceedings-of-spie on 26 Apr 2023 Terms of Use: https://www.spiedigitallibrary.org/terms-of-use 


\title{
Conference Committee
}

\author{
Symposium Chairs
}

Halina Rubinsztein-Dunlop, The University of Queensland (Australia)

Mark L. Brongersma, Geballe Laboratory for Advanced Materials

(GLAM), Stanford University (United States)

Symposium Co-chairs

Harry A. Atwater Jr., California Institute of Technology (United States)

Nikolay I. Zheludev, Optoelectronics Research Centre

(United Kingdom) and Nanyang Technological University

(Singapore)

\section{Conference Chairs}

Ganapathi S. Subramania, Sandia National Laboratories (United States)

Stavroula Foteinopoulou, The University of New Mexico (United States)

\section{Conference Program Committee}

Andrea Alù, The City University of New York (United States)

Koray Aydin, Northwestern University (United States)

Paul V. Braun, University of Illinois at Urbana-Champaign (United States)

Che Ting Chan, Hong Kong University of Science and Technology (Hong Kong, China)

Zhigang Chen, Nankai University (China) and San Francisco State University (United States)

Dmitry N. Chigrin, RWTH Aachen Universität (Germany)

Shanhui Fan, Stanford University (United States)

Didier Felbacq, Université Montpellier (France)

Joseph W. Haus, University of Dayton (United States)

Stephen Hughes, Queen's University (Canada)

Boubacar Kante, University of California, San Diego (United States)

A. Femius Koenderink, AMOLF (Netherlands)

Alexander V. Kildishev, Purdue University (United States)

Nathaniel Kinsey, Virginia Commonwealth University (United States)

Yuri S. Kivshar, The Australian National University (Australia)

Cefe López, Consejo Superior de Investigaciones Científicas (Spain)

Liam O'Faolain, Cork Institute of Technology (United Kingdom) and Tyndall National Institute (Ireland)

Rupert F. Oulton, Imperial College London (United Kingdom) 
Nicolae-Coriolan Panoiu, University College London (United Kingdom)

Michelle L. Povinelli, The University of Southern California (United States)

Christophe Sauvan, Laboratoire Charles Fabry (France)

Jörg Schilling, Martin-Luther-Universität Halle-Wittenberg (Germany)

Gennady B. Shvets, Cornell University (United States)

Volker J. Sorger, The George Washington University (United States)

Andrey A. Sukhorukov, The Australian National University (Australia)

Kosmas L. Tsakmakidis, Ecole Polytechnique Fédérale de Lausanne (Switzerland)

Georgios Veronis, Louisiana State University (United States)

Daniel M. Wasserman, The University of Texas at Arlington (United States)

Sharon M. Weiss, Vanderbilt University (United States)

Session Chairs

1 Novel Nonlinear Photonic Phenomena I

Michael Scalora, U.S. Army Aviation and Missile Command (United States)

2 Novel Nonlinear Photonic Phenomena II

Igal Brener, Sandia National Laboratories (United States)

3 New Platforms for Harnessing and Probing Thermal Effects

Thomas Taubner, RWTH Aachen Universität (Germany)

4 Phase-Change Materials for Tuneable Photonics

Simeon Bogdanov, Purdue University (United States)

5 Photonics with Atomically Thin Materials I

Joshua D. Caldwell, Vanderbilt University (United States)

6 Photonics with Atomically Thin Materials II

Dmitry N. Chigrin, RWTH Aachen Universität (Germany)

7 Topological and Non-Reciprocal Photonics I

Stephen Hughes, Queen's University (Canada)

8 Twisted Light: Orbital Angular Momentum and Vortex States

Marin Soljacic, Massachusetts Institute of Technology (United States)

9 Topological Photonics in Non-Hermitian and Non-Linear Systems I

Avadh B. Saxena, Los Alamos National Laboratory (United States) 
10 Topological and Non-Reciprocal Photonics II

Sander A. Mann, The University of Texas at Austin (United States)

11 Topological Photonics in Non-Hermitian and Non-Linear Systems II Hugo Doeleman, AMOLF (Netherlands)

12 Advanced Tunable and Dynamic Platforms with Structured Materials Philippe Tassin, Chalmers Universitet of Technology (Sweden)

13 Harnessing Light-Matter Interaction I

Alexander Quandt, University of the Witwatersrand, Johannesburg (South Africa)

14 Photonic Memories and Neuromorphic Computing

Ganapathi S. Subramania, Sandia National Laboratories (United States)

15 Non-Hermitian Photonics and PT-Symmetric Systems

Avadh B. Saxena, Los Alamos National Laboratory (United States)

16 Addressing Modeling Challenges in Emerging and Active Photonic Systems

Stavroula Foteinopoulou, The University of New Mexico (United States)

17 Enhancing and Measuring Optical Forces

Femius Koenderink, AMOLF (Netherlands)

18 New Materials for Photonics: From "Nature's Lab" to Advanced Fabrication

Romuald Houdré, Ecole Polytechnique Fédérale de Lausanne (Switzerland)

19 Harnessing Light-Matter Interaction II

Raktim Sarma, Sandia National Laboratories (United States) 
Proc. of SPIE Vol. 10721 1072101-10

Downloaded From: https://www.spiedigitallibrary.org/conference-proceedings-of-spie on 26 Apr 2023 Terms of Use: https://www.spiedigitallibrary.org/terms-of-use 


\section{Introduction}

Modern photonic technologies require extreme light control capabilities in the full four dimensions (4D) of space and time, well beyond what natural materials can offer. Nowadays, very sophisticated structured material platforms are possible thanks to the rapid advancement of nanofabrication tools and methods. These were catalysts for enabling exotic photonic behaviors opening up avenues and breaking boundaries in a vast spectrum of technologies such as sustainable energy, sensors, medical diagnostics or information transfer and computing. The capabilities of passive platforms comprising metals or dielectrics transcend into new unchartered domains when paired-up with active or tunable material, as for example gain media, non-linear optical materials, phase-change materials, graphene and atomically thin semiconductors, magneto-photonic media, as well as quantum emitters. The fourth dimension, time, has emerged as a key "tuningknob" for molding the behavior of light; dynamic photonic platforms are central in current research activities in the community.

The Active Photonic Platforms X marks the $10^{\text {th }}$ installment of a conference series that sprung out of an SPIE conference convened by Paul Braun (UIUC) and Sharon Weiss (Vanderbilt U.) featuring active and tunable responses in photonic crystals [Tuning the Optical Response of Photonic Bandgap Structures III (2006)]. Over the years, this conference series, initially named Active Photonic Crystals but re-named to Active Photonic Materials and finally to Active Photonic Platforms in 2017, has evolved to snapshot the rapidly evolving research developments related to active control of light propagation and photonic responses. A range of topics involving active, dynamic, and tunable control of light in a plethora of structured-material platforms as well as systems involving atomically thin materials have been presented in this year's event. Novel behaviors for light waves in passive platforms that would enable new research directions in active photonics have also been featured. Below, we give a bird's eye view of the fascinating research presented in Active Photonic Platforms X.

Extra-ordinary non-linear light control was a central theme in the conference this year, with the conference opening with two related sessions. The opening keynote presentations of these sessions reported cutting-edge research on unprecedented light manipulation with accelerating beams which could become a paradigm shift in all-optical circuit applications [Presentation 10721-4] or give rise to exotic nonlinear phenomena such as non-integer harmonic generation [Presentation 107211]. Moreover, an intriguing keynote presentation [Presentation 10721-3] showed how a metasurface can lead to unconventional frequency mixing capabilities opening new pathways for control of non-linear optical processes. A common thread in the talks in the first two session were innovative approaches to enhancement of non-linear effects which was demonstrated by utilizing epsilonnear-zero (ENZ) materials [Presentations 10721-4, 10721-5], nanoplasmonic 
waveguides [Presentation 10721-2], nano-antennae [Presentation 10721-8], metasurfaces [10721-3] or even photonic-crystal cavities (as shown by Presentation 10721-80 in a subsequent session). Theoretical developments to understand the impact of interfaces on the non-linear optical parameters of a material have also been presented [10721-6] which are extremely important in guiding the design of nanoscale systems and heterostructures for non-linear optics.

As structures are brought to the nanoscale spatial monitoring of optically induced thermal responses are key to characterizing device stability and performance. Di Wang of Purdue Univ. [Presentation 10721-12], one of the Best student Presentation award recipients, reported on a new method to map the spatial temperature distribution of a plasmonic system. Moreover, Presentation 10721-13 utilized a controlled light-to-heat conversion for biomolecular sensing. In addition, many presentations focused on absorption and thermal-emission management towards efficient infrared sources by utilizing phonon-polariton materials [Presentations 10721-9, 10721-10, 10721-11], with Presentation 10721-9 reporting on the potential of active tunability/modulation of these effects with free-carrier injection. Presentation 10721-15 presented an alternative approach to tailoring extraordinary thermal emission by incorporating phase-change materials. As this exciting invited talk reported, these materials can evoke an unexpected thermal emission signature owing to a temperature induced phase-transition which makes them suitable coatings for thermal camouflage.

Actually, phase-change (phase-transition) materials, i.e. materials that can change their structural and electronic properties with temperature, have recently emerged as a key material component in photonic platforms. Because of their dynamically tunable photonic responses these materials are gaining increasing interest for photonic applications. Presentation 10721-18 reported the development of new classes of phase-change materials having in particular photonic applications in mind; phase-change materials used in photonics are typically "borrowed" from widely used materials in electronics which tend to be rather lossy when interacting with visible light. Platforms utilizing phase-change materials were also shown to lead to tunable color reflection filters [Presentation 10721-16] or switchable one-way reflection suppression [Presentation 10721-17]. Moreover, Presentation 10721-14 introduced the amazing possibility to address locally the resonances of individual antennae on an array by local change of the phase of the material environment from amorphous to crystalline. The results reported by this invited presentation would open-up a whole new area of photonic platforms with a programmable response.

Novel photonic platforms for memories and computing was actually an emergent topic in the conference with exciting talks reporting on architectures that could become trend-setters in the years to come. Phase-change-materials were also central in this direction with a cross-cutting invited talk [Presentation 10721-59] reporting on the possibility of related photonic chips for arithmetic operations. Furthermore, two more invited talks reported forefront research on how to exploit 
thermo-optic or electro-optic effects to construct memory devices [Presentation 10721-56] or highly energy efficient (a J/bit) modulators for neuromorphic photonic compute engines [Presentation 10721-57]. Integrated neuromorphic photonics could revolutionize modern computing; an invited presentation [Presentation 10721-58] reported on the breakthrough possibilities of these platforms but also the important challenges to be met.

Furthermore, Presentation 10721-7 envisioned a synthetic multidimensional spectral space constructed from all-optical spectral reshaping in a non-linear fiber which could be utilized for quantum computations. A compelling keynote presentation [10721-37] also showed how a judiciously designed photonic-crystal slab in transmission mode effectively behaves as the Laplacian operator opening new possibilities for leading-edge technologies in image processing. Beyond photonicbased computing platforms, a groundbreaking keynote presentation [Presentation 10721-40] suggests that certain complex scientific problems could be solved without actually performing the relevant calculations. This fascinating talk showed that artificial intelligence (Al), similar to deep-learning algorithms currently used to train neural networks e.g. for image recognition, can predict the photonic band structure of a certain photonic crystal structure (and vice-versa); astonishingly, the Al predictions fall extremely close to the actual calculations.

Just like phase-change materials, 2D and quasi-2D materials after blooming in the electronics world have well entered the world of photonics and the research of their responses to light is rapidly expanding. In addition to presentations throughout, two focus sessions on "photonics with atomically thin materials" were intended to capture recent advances at the forefront of this sub-area. Bridging across the 2D materials and phase-change materials domains, invited Presentation 10721-77 discussed examples and avenues by which certain atomically thin materials can operate as phase-transition materials. Moreover, an engaging invited presentation showed the realization of high quality lateral heterostructures comprising twodimensional transition metal dichalcogenide (2D TMDC) for optoelectronic applications [Presentation 10721-20]. Furthermore, exciting talks underscored the vast potential of $2 \mathrm{D}$ materials for photonics reporting on atomically thin hyperbolic materials enabled by anisotropic phonon-polaritons [Presentations 10721-19], modelling and tunability of graphene and 2D material plasmons [Presentations 10721-21 and 10721-23] as well as DC-current-induced asymmetric plasmon propagation in graphene [Presentation 10721-22].

One of the keynote presentations [Presentation 10721-19] also delved into entirely unexplored Physics in these systems reporting on the ultrafast dynamics of photoexcited plasmons in graphene as well as long range plasmon propagation at cryogenic temperatures. Moreover, given the extra-ordinary photonic responses of 2D materials, platforms that interface them with structured materials and/or quantum emitters have naturally piqued scientific curiosity. Many exciting presentations [Presentations 10721-25, 10721-26, 10721-27 and 10721-81] reported research to better understand the behavior of quantum emitters in atomically thin 
materials for optoelectronic applications. Furthermore, a keynote presentation [Presentation 10721-24] brought about transformative research in random lasing applications by utilizing MXene flakes in a solution with a gain material. This fascinating talk showed that the random MXene-flake medium offers unprecedented control over the feedback mechanism owing to the non-linear optical responses of the individual MXene nanoflakes.

Despite years of research the interaction of emitters with structured or novel material environments actually still remains in the center of scientific inquisition with adventurous paths still to explore. Many interesting talks reported on advances in the area of harnessing light-matter interactions. An intriguing invited talk [Presentation 10721-53] discussed how the quality factor of certain resonators should be manipulated so that to optimize interaction with quantum emitters; the trivial widespread assumption the higher-Q the better may not necessarily hold. Moreover, Presentation 10721-60 reports that conventional wisdom from the Purcell expression may not apply at exceptional points of degeneracy, which yield higher than predicted spontaneous emission rates. Furthermore, for localized-plasmon resonances Presentation 10721-79 analyzed and experimentally demonstrated the different regimes of interaction from enhancement of spontaneous emission to the formation of hybrid plasmon-exciton modes. When interacting with active media, Presentation 10721-82 showed that the geometric characteristics of Mie resonators impact the lasing frequency, while Presentation 10721-55 demonstrated spontaneous emission enhancement in a particular implementation of Mie resonators. The crucial parameter controlling light-matter interaction in these systems is the enhanced power of the electric field. Presentation 10721-54 develops a sum-rule that provides a limitation on the associated power-bandwidth product.

Also, several engaging presentations reported on tunable EM effects and reconfigurable platforms [presentations 10721-47, 10721-48, 10721-49, 10721-50, 10721-51, 10721-76]. The underlying mechanisms relied on incorporating voltage tunable material, or material tunable with optical pumping which are interfaced with ring resonators or metasurfaces. For example, Ghazaleh Kafaie Shirmanesh of Caltech [Presentation 10721-49], also one of the Best student paper award recipients, presented a reconfigurable beam steering metasurface operating at telecommunication wavelength. The reconfigurability in this case is enabled by incorporating ITO which has voltage-tunable optical properties.

Material structuring in the 3D space together with time-modulated material properties constitutes full 4D control of light in space and time and can lead to non-reciprocal photonic effects as was reported by Presentations 10721-4, 1072129 and 10721-37. Aside from time-modulation, a captivating keynote presentation [10721-30] reviewed the different mechanisms to non-reciprocity and presented a novel possibility of an optical diode effect by employing a specially oriented polar ferromagnet. Non-reciprocal effects by employing non-linearities [10721-29] and magnetophotonic effects [10721-31] have also been reported. Presentation 1072132 also showed that a heterogeneous magnetic field of opposite sign in different 
regions essentially creates an artificial boundary in an electron gas supporting topological edge-states.

Topological and non-Hermitian photonics continued to be central in focus in this year's conference with related effects featured over five sessions. Many fascinating presentations covered a range of innovative approaches to extreme light control. Photonic graphene paradigms of topological edge states have been presented by Presentations 10721-41 and 10721-43 while Presentation 10721-38 discussed the presence of photonic topological phases under non-linearity and gain. Topological type of sources and lasing have also been reported [Presentation 10721-44 and 10721-45]. The behavior of systems around exceptional points was discussed by Presentation 10721-62 emphasizing that although these have been traditionally associated with non-Hermitian PT-symmetric systems they can be present also in lossless systems such as coupled waveguides. PT-symmetric system for reconstruction of mode-coherence [Presentation 10721-61], for uni-directional reflection suppression [Presentation 10721-39] and asymmetric scattering in gainloss nanoparticle dimers [Presentation 10721-63] have also been reported.

Furthermore, a key outstanding problem in optics and photonics is how to create and control the angular momentum of light. Progress in this area will also trigger breakthrough capabilities in active photonics. A captivating keynote presentation [Presentation 10721-33] opening the related "twisted light" focus session presented a framework based on symmetry to understand how chiral matter can engage with structured light that has an orbital angular momentum. Then, a fascinating invited presentation [Presentation 10721-36] showed how twisted light can emerge from plasmonic nanoantennae, while Presentation 10721-34 presented how to create vector vortex beams and discussed devices to detect them. Moreover, Hugo Doeleman of AMOLF [Presentation 10721-35], who is also one of the recipients of the Best student Presentation award, reported on the first-time experimental observation of a polarization vortex emerging from a bound state in the continuum.

Numerous extra-ordinary optical effects and platforms were reported in the conference. While all these interesting structures arise from man-made architectures, we note in passing that extraordinary photonics can emerge also from naturally evolved structures. For example, Presentation 10721-73 discussed how plant tissue has evolved in different species to control the influx of light for photosynthesis. It should also be noted that a key important aspect in advanced photonic systems both for the stability of practical systems but also for the capability to manipulate nanoscale objects is that of Casimir forces and optical forces. A related session and stimulating invited talks focused on bringing out new Physics and fundamental understanding on these forces in different systems. In particular, theoretical analysis or experiments on Casimir and/or optical forces were reported for artificially patterned surfaces [Presentation 10721-70], metamaterials [Presentation 10721-71] and gold-coated microspheres [Presentation 10721-72]. 
Of course, all these fantastic extra-ordinary photonic phenomena could not have been envisioned or realized without progress in associated theory, methodology, and numerical techniques. There were a few talks and a dedicated session to highlight important theory advances for the study of extra-ordinary photonic effects. In particular, Presentation 10721-75 borrowed from photonic-band-gap theory in dielectric structures to design a reflector comprising a single layer of a metallic nanoparticle array. An engaging and instructive invited talk [Presentation 10721-69] presented a framework to understand and design extra-ordinary scattering regimes for structures made from topological insulator material. Another interesting invited presentation [Presentation 10721-67] emphasized the need for atomistic-level calculations for modelling the responses in certain plasmonic systems which are not adequately captured by more widespread classical macroscopic approaches. Furthermore, a cross-cutting presentation [Presentation 10721-68] discussed how methods must be brought together in a multi-physics framework. In particular, this presentation discussed the need to couple electromagnetic and heat transfer solvers self-consistently in order to tackle the response of photonic platforms with phase-change materials. As photonic systems become more sophisticated with key operating principles crossing disciplinary boundaries the development and advancement of multi-physics frameworks is absolutely crucial to enable breakthrough phenomena and technologies.

Last, but not least, we would like to highlight three most challenging problems in theory and modelling of passive and active photonics that are related to highimpact application domains. The first one is a theoretical framework to describe modes of lossy resonators, such as plasmonic nanoparticles, to capture the behavior of quantum emitters in their vicinity. The second one is a framework that can predict radiation losses in membrane geometries relevant to slow-light and topological edge-state waveguides in planar photonic crystal systems. An incisive keynote presentation [Presentation 10721-52] presented approaches to these challenging problems which can catalyze progress in the related areas offering design guidance for high performing practical designs.

The third challenging theory problem relates to a framework for structured materials with gain media. Widespread rather common treatments of gain media involve models that use a complex, frequency dispersive, refractive index. However, such models miss out completely on the dynamic evolution of the gain media responses to the incident field. Two informative and engaging talks [1072164 and 10721-65] presented the recent progress of multi-physics modelling of different types of gain material in time-domain. The treatment discussed in these talks couples specific atomic transition models for the behavior of the gain medium with exact full-wave EM simulations in time-domain. The talks presented examples of application of this multi-physics framework in areas of high current interest in active photonics, such as nanolasing and reverse saturable absorption. 
Along with the talks recapped above, our conference also included a lively poster session with themes related to graphene photonics [Presentations 10721-84, 1072186] and information storage with optical beams [Presentations 10721-85, 10721-87] complementing on the topics of the oral presentations.

While recapping the conference presentations, we mentioned in passing the three winners of the best student Presentation competition that our conference ran. The award recognizes the best contributed presentations that were presented by a student author. For this year, there were three finalist winners that have tied in first place. We would like to extend our warm congratulations to the best student Presentation winners! We would also like to take this opportunity and thank all student contributors for their enthusiasm with which they participated in this competition, giving amazing talks and reporting on incredibly interesting research.

The winners of the student award competition were selected by a jury comprising the program committee members and expert attendees in the conference in a two-phase process. The first phase of the competition is based on the abstract submissions and lead to the selection of six contributions which competed further on-site based on the oral presentation. The three winners that tied in first place were recognized with an SPIE award certificate at the "Award Announcement," session on the last day of the conference. Also, SPIE publicity covered this session with a highlight on the 2018 Best student Presentation award winners in the conference website. We enlist below again the winning Presentations with the student author's name being designated with a bold font.

Presentation 10721-12: Mapping temperature distribution of optically pumped gap plasmon structure using thermoreflectance imaging

Author(s): Di Wang, Kerry Maize, Yee Rui Koh, Purdue Univ. (United States); Maowen Song, Purdue Univ. (United States), Chongqing Univ. (China); Alexandra Boltasseva, Purdue Univ. (United States), Technical Univ. of Denmark (Denmark); Vladimir M. Shalaev, Ali Shakouri, Alexander V. Kildishev, Purdue Univ. (United States)

Presentation 10721-35: Experimental observation of a polarization vortex at a bound state in the continuum

Author(s): Hugo Doeleman, AMOLF (Netherlands), Univ. van Amsterdam (Netherlands); Francesco Monticone, Cornell Univ. (United States); Wouter den Hollander, Femius Koenderink, AMOLF (Netherlands); Andrea Alù, The Univ. of Texas at Austin (United States)

Presentation 10721-49: Reconfigurable beam steering metasurface at telecommunication wavelengths

Author(s): Ghazaleh Kafaie Shirmanesh, Ruzan Sokhoyan, Pin Chieh Wu, Harry A. Atwater, Caltech (United States) 
Active Photonic Platforms $X$ brought together theorists and experimentalists to exchange state-of-the art results in a rapidly evolving area of research. As conference chairs, we would like to thank our program committee members, the session chairs as well as members of the best student Presentation award jury for contributing in numerous ways to the success of this conference. Last but not least, our sincere thanks go to all the authors of the 10721 conference who engaged with their attendance and questions, presentations as well as manuscripts making this conference a truly stimulating and vibrant event!

\section{Ganapathi S. Subramania Stavroula Foteinopoulou}

